\title{
Polarization-resolved nonlinear dynamics induced by orthogonal optical injection in long-wavelength VCSELs
}

\author{
P. Pérez, A.Quirce, L. Pesquera, and A.Valle
}

\begin{abstract}
We report a first experimental study of the polarization-resolved nonlinear dynamics of a $1550 \mathrm{~nm}$ singlemode VCSEL subject to orthogonal optical injection. Stability maps identifying the boundaries between regions of different nonlinear dynamics are reported for two different values of the bias current. A rich variety of nonlinear behaviours including periodic, period doubling, and irregular dynamics are observed for both polarizations. Polarization switching and injection locking induced by the optical injection are also included in the mapping. Analysis is given also in terms of the frequency detuning between the injected light and the orthogonal linear polarization of the VCSEL. When the frequency detuning is positive polarization switching can be observed in a periodic dynamical regime, including both period one and period two behaviors. For positive frequency detuning, the only polarization that contributes to the dynamics of the total power is the orthogonal polarization. For negative frequency detunings both linear polarizations contribute to the dynamics of the total power.
\end{abstract}

Index Terms-Polarization switching, optical injection, injection locking, nonlinear dynamics, vertical-cavity surfaceemitting lasers (VCSELs).

\section{INTRODUCTION}

$\mathrm{O}$ ptical injection in semiconductor lasers is a technique that is commonly employed to improve the performance of these lasers without modifying their design [1-3]. It can be used for reduction of the laser linewidth, of the mode partition noise or for modulation bandwidth enhancement. Interest in optical injection in vertical-cavity surface-emitting lasers (VCSELs) has recently increased [4-8] due to the inherent advantages of this type of semiconductor lasers. These include singlelongitudinal mode operation, circular beam profile, low threshold current, reduced fabrication costs, ease of fabrication of 2D arrays, compactness, etc. [4]. Besides the interest from

Manuscript received November, 2010. This work has been funded in part by the Ministerio de Ciencia e Innovación, Spain, under project TEC200914581-C02-02, and by EC Project PHOCUS (FP7-ICT-2009-C-240763).

P.Pérez, A.Quirce, L. Pesquera and A.Valle are with the Instituto de Física de Cantabria, Consejo Superior de Investigaciones Científicas (CSIC)Universidad de Cantabria, E-39005 Santander, Spain. P. Pérez and A. Quirce are also with the Departamento de Física Moderna, Univ. de Cantabria, Facultad de Ciencias, E-39005, Santander, Spain (Phone: (34) 942201465. Fax: (34) $942 \quad 200935 . \quad$ E-mail: perezg@ifca.unican.es; quirce@ifca.unican.es; pesquerl@ifca.unican.es); valle@ifca.unican.es the applications point of view, optical injection in semiconductor lasers is interesting for obtaining a wealth of complex nonlinear dynamics and bifurcations [9]. Period doubling, quasiperiodicity, chaos and injection locking have been demonstrated [9]. These studies have also been extended to VCSELs [10-17] since they offer additional degrees of freedom, like the direction of the emitted polarization and the presence of multiple transverse modes, when compared with their edge-emitting counterparts. Extensive research efforts have been undertaken to explain the polarization switching (PS) in free-running VCSELs [18-22]. Different physical mechanisms can lead to PS, such as the relative modification of the net modal gain and losses with the injection current [1819], spatial hole burning [20], or microscopic spin-flip processes in the presence of birefringence and linewidth enhancement factor [21]. In all the previous mechanisms the role played by birefringence and dichroism [22] is essential. Most of the studies about the nonlinear dynamics of optically injected VCSELs have been performed for devices emitting near $850 \mathrm{~nm}$ wavelength. The behaviour of these systems not only includes high-frequency periodic dynamics, chaotic dynamics and injection locking, but also PS and Optical Bistability (OB). The extension of these results to the telecom wavelength of $1550 \mathrm{~nm}$ is of interest in present and future optical telecommunication networks. For instance, applications of these phenomena for all-optical signal processing using VCSELs has recently appeared [3-4],[8].

Although pionering experiments considered parallel polarization directions for injected and VCSEL laser beams [10], most of the studies on dynamics have been performed under orthogonally polarized optical injection conditions. Orthogonal optical injection (OOI) was first proposed by Pan et al. to obtain PS and OB between the two linear polarizations of a short-wavelength VCSEL [23]. In this configuration linearly polarized light from an external laser is injected orthogonally to the linear polarization of a free-running VCSEL. PS and OB induced by OOI have been also recently obtained in 1550-nm wavelength VCSELs [24-28].

Most of the nonlinear dynamics analysis have been done in short-wavelength VCSELs with small values of the birefringence and showing PS in absence of optical injection. Rich nonlinear dynamics have been found including period doubling, quasi-periodicity, injection locking, bistability and chaos [13-16]. A mapping of the dynamics identifying boundaries between those behaviors was presented in [13]. A Hopf bifurcation, not reported for edge-emitting lasers, on a 
two-polarization-mode solution delimits the injection locking region [14]. A torus bifurcation, that corresponds to the excitation of two-polarization mode dynamics in the route to PS and injection locking, has been described theoretically and experimentally [14].

Only recently the nonlinear dynamics of a $1550-\mathrm{nm}$ wavelength single transverse mode VCSEL subject to OOI has been experimentally analyzed [17]. This VCSEL was characterized by very large values of the birefringence parameter and by emission in a single linear polarization over the whole bias current range in absence of optical injection. A stability map was reported for the total power identifying the boundaries between regions of different behavior, including periodic dynamics, PS and irregular and possibly chaotic behaviour [17]. However that work only reported the analysis of the dynamics of the total power because only measurements of their RF spectra and temporal traces were performed.

In this work we report for the first time to our knowledge an experimental study of the polarization-resolved nonlinear dynamics of a $1550 \mathrm{~nm}$-VCSEL subject to OOI. Our VCSEL is characterized by very large values of the frequency splitting between both linear polarizations. The device emits in a linearly polarized fundamental transverse mode over the whole bias current range. Measured stability maps identifying the boundaries between regions of different nonlinear dynamics are reported for several values of the bias current. A rich variety of nonlinear behaviours including periodic, period doubling, and irregular dynamics are observed for both polarizations. More diverse dynamics is observed as the bias current is increased. Polarization switching and injection locking induced by the optical injection are also discussed. We also make an analysis for different frequency detunings between the injected light and the orthogonal linear polarization of the VCSEL. We show that the contribution of the linear polarizations to the dynamics of the total power depends on the sign of the frequency detuning.

The paper is organized as follows. Section II describes the experimental setup. Nonlinear dynamics for small and large values of the bias current are analyzed in sections III and IV, respectively. Finally, in section V, a summary and conclusions are presented.

\section{EXPERIMENTAL SETUP}

The orthogonal optical injection from a tunable laser (Tunics Plus-CL) into a quantum-well $1550 \mathrm{~nm}$ VCSEL is achieved by using the all-fibre setup shown in Fig. 1. A commercially available VCSEL (Raycan ${ }^{\mathrm{TM}}$ ) was used in the experiments. The same device was also analyzed in [17]. The VCSEL bias current and temperature are controlled, respectively, by a laser driver (Thorlabs LDC200) and a temperature controller (Thorlabs TED200). The temperature was held constant at $298{ }^{\circ} \mathrm{K}$ during the experiments. A variable optical attenuator is included after the tunable laser to control the level of optical power of the externally injected signal. The output of the tunable laser is then injected into the VCSEL using a three-port optical circulator. The polarization of the external signal is controlled using a fibre polarization controller. A 90/10 fibre directional coupler divides the optical path in two branches; the $10 \%$ branch is used to monitor the optical input power with a power meter whereas the $90 \%$ output is directly connected to the SL. The reflected output of the VCSEL is analyzed by connecting different measurement equipment to the third port of the circulator. One half of the power is used to obtain optical spectra with an Optical Spectrum Analyzer (OSA) or with a Fabry-Perot (FP) analyzer with resolutions of 70 and $4 \mathrm{pm}$, respectively. The other half of the power is directed to a polarization beam splitter that selects the polarization direction in which the RF spectra are measured. A fast-photodiode (9 GHz bandwidth) and an electrical spectrum analyzer (Anritsu MS2719B) were used to obtain the RF spectra.

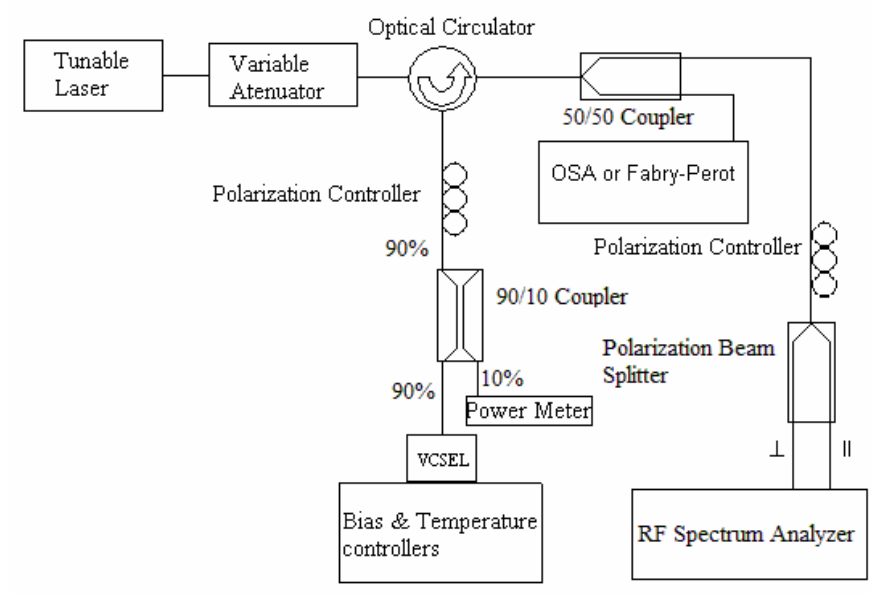

Fig. 1. (a) Experimental setup for orthogonal optical injection in a VCSEL.

Fig. 2(a) plots the L-I curve of the free-running device. The VCSEL emits in the fundamental transverse mode with a threshold current of $I_{t h}=1.64 \mathrm{~mA}$. The VCSEL emits in a linear polarization which we will call the "parallel" polarization. Emission in the parallel polarized fundamental mode is obtained along the whole current range. Fig. 2(b) shows the optical spectrum of the VCSEL biased at $8 \mathrm{~mA}\left(4.88 I_{t h}\right)$. The lasing mode of the device with parallel polarization is located at the wavelength $\lambda_{\|}=1536.6 \mathrm{~nm}$. The subsidiary mode corresponds to the fundamental transverse mode with "orthogonal" polarization and its wavelength $\left(\lambda_{\perp}\right)$ is shifted $0.49 \mathrm{~nm}$ to the long-wavelength side of the lasing mode. This value for the frequency splitting between the two orthogonal polarizations is very large in comparison to those reported in short-wavelength devices [13]. A Side Mode Suppression Ratio (SMSR) of $43 \mathrm{~dB}$ is measured for the orthogonal polarization. Spectra of this form are measured for all biases and no polarization switching is observed for bias current above the threshold value. This is probably due to a large value of the dichroism parameter for our VCSEL. 


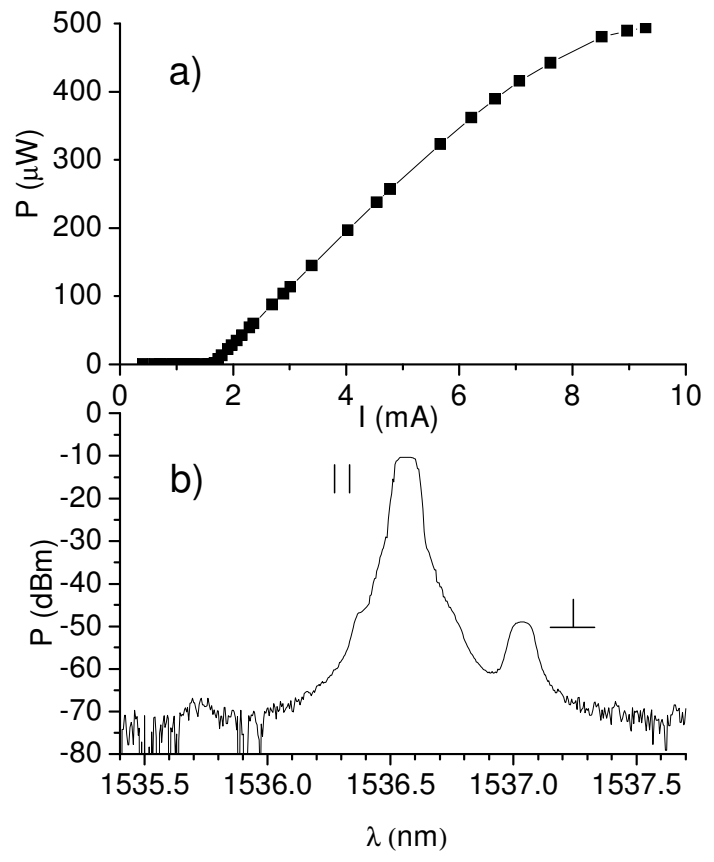

Fig. 2. (a) L-I curve and (b) spectrum of the free- running VCSEL (applied bias current of $8 \mathrm{~mA}$ ) [17].

\section{NONLINEAR DYNAMICS AT SMALL BIAS CURRENT}

Fig. 3 shows the mapping of the dynamics of the total power of the VCSEL when it is subject to orthogonal optical injection. Measurements are performed with the VCSEL biased with an applied current of $4 \mathrm{~mA}\left(2.44 I_{t h}\right)$. The relaxation oscillation frequency of the free-running VCSEL, $v_{R}$, is $2.95 \mathrm{GHz}$. The optical injection is characterized by its strength, given by the value of the optical power arriving at the VCSEL, $P_{i n j}$, and by its frequency, $v_{i n j}$. We consider values of $v_{i n j}$, that are close to the frequency of the perpendicular polarization, $v_{\perp}$, the frequency detuning being $\Delta v=v_{i n j}-v_{\perp}$. The experimental mapping is obtained by fixing a value of $\Delta v$ and increasing $P_{i n j}$ from low values. The different dynamical regimes of the total power are then identified by using its RF spectrum, recorded at the output of the 50/50 coupler. In Fig. 3 the region P1 shows periodic (period 1) behaviour, region IR corresponds to irregular and possibly chaotic dynamics, and region SL represents the stable locking range. We have considered P1 dynamics when the peak in the RF spectrum measured in the $\mathrm{RF}$ analyzer is $10 \mathrm{~dB}$ above the noise floor. The shape of the $\mathrm{SL}$ region is rather symmetric around $\Delta v=0$, in contrast to the asymmetric shape observed in short-wavelength VCSELs [13]. In fact stable locking associated to the fundamental transverse mode was only obtained at negative values of $\Delta v$ [13]. Also in contrast with the results reported in [13], the irregular behavior observed in Fig. 3 is only obtained for negative values of $\Delta v$ and is not the result of a period doubling route to chaos. All these differences are possibly due to the very large value of the birefringence parameter and to the single polarization mode characteristics of our free-running long-wavelength VCSEL.

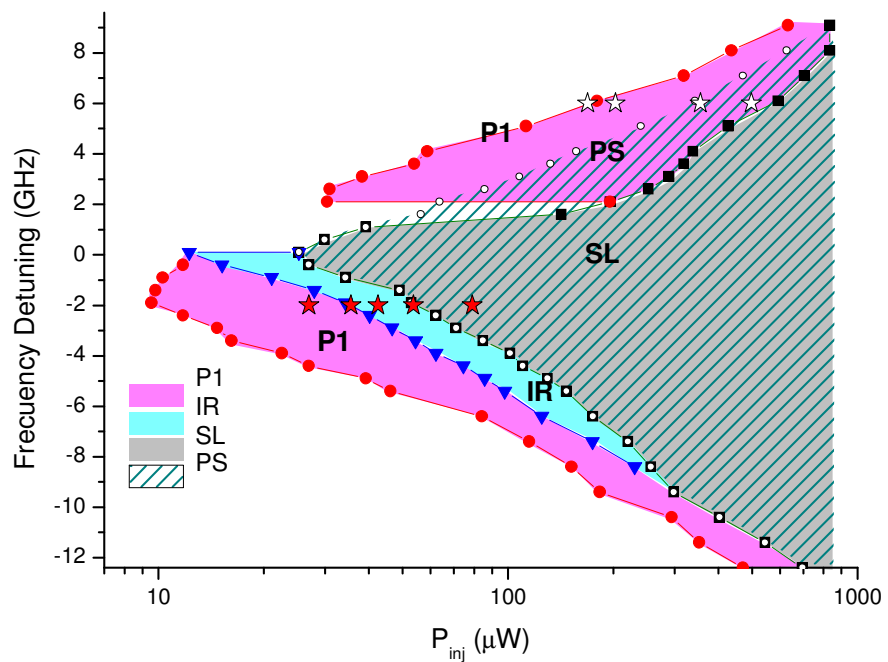

Fig. 3. (color online) Stability map of the VCSEL subject to orthogonal optical injection [17]. Different regions are observed: SL (stable injection locking), P1 (period 1), IR (irregular dynamics), and PS (polarization switching). The stars mark the situations analyzed in Figs 4 and 5. Applied bias current of $4 \mathrm{~mA}$

Fig. 3 also shows the injected power required for PS to the orthogonal polarization of the VCSEL. It is found that PS is always accompanied with stable locking for negative $\Delta v$. However, PS can be also observed in a periodic (P1) dynamical regime for positive values of $\Delta v$. RF spectra for the two polarizations and for the total power are shown in the left column of Fig. 4 for different dynamical regimes. They correspond to a fixed frequency detuning of $6 \mathrm{GHz}$ and different values of the injected power. The cases considered in Fig. 4 are identified in Fig. 3 with white stars. The corresponding optical spectra of the total emitted light obtained with the FP analyzer are shown in the right column of Fig. 4. Showing these results is enough to describe the optical spectra of both linear polarizations. We have checked that optical spectra of the orthogonal (parallel) polarization correspond to the optical spectra of the total emitted light for frequencies below (above) $30 \mathrm{GHz}$. The zero value of the frequency has been chosen to coincide with the frequency of the orthogonal polarization without optical injection. The values of the frequency of the optical injection are marked with arrows in the plots corresponding to optical spectra.

Fig. 4(a) show the dynamics just before reaching the P1 region. The RF spectra shown in this work have been obtained by subtracting the RF spectra in the absence of light to the RF spectra with optical injection. We have done it in order to subtract the noise in the photodetector and RF analyzer. A peak in the RF spectrum of the total power appears near the frequency detuning. A similar peak appears in the RF spectrum of the orthogonal polarization power. The optical spectrum of Fig. 4(b) shows that two peaks are excited. The highest one corresponds to the injected light that is reflected at the VCSEL cavity while the smallest one corresponds to the parallel polarization mode of the VCSEL. The parallel polarization is excited with a constant value because its spectrum is flat as it 
is shown in Fig. 4(a).
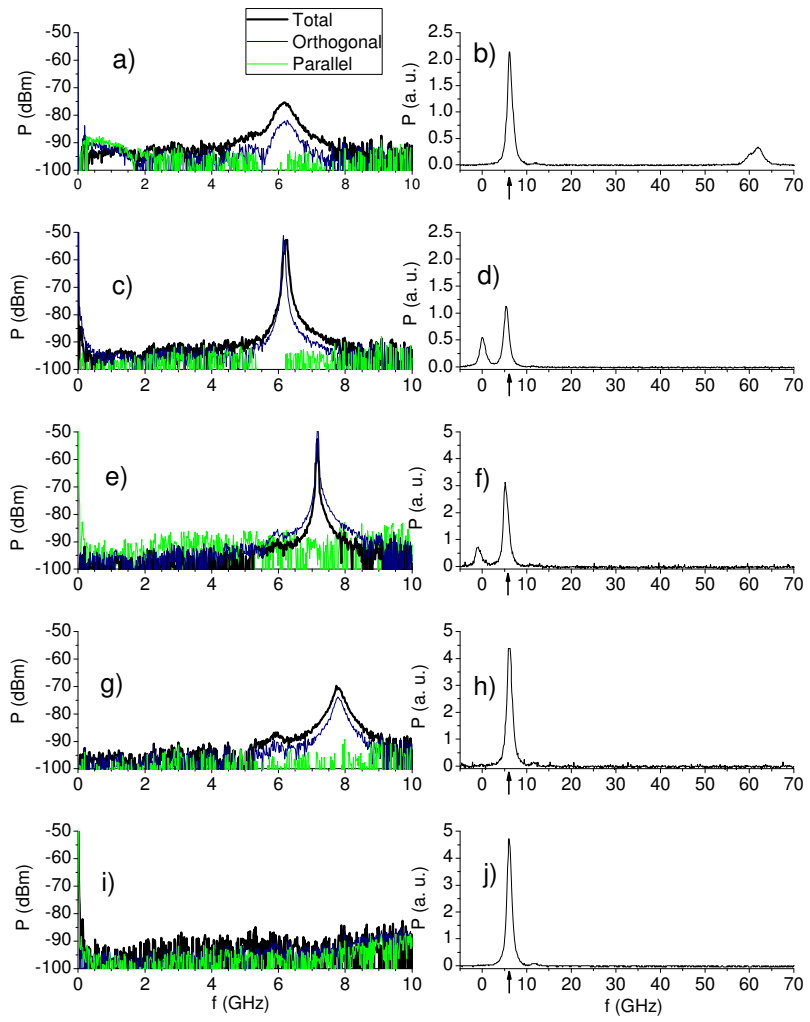

Fig. 4. (color on line) (Left column) RF spectra of the total and polarized powers. (Right column) Optical spectra of the total emitted light. Several values of injected power are considered: (a,b) $P_{i n j}=168.9 \mu \mathrm{W},(\mathrm{c}, \mathrm{d}) P_{i n j}=$ $202.7 \mu \mathrm{W}$, (e-f) $P_{i n}=355.1 \mu \mathrm{W},(\mathrm{g}, \mathrm{h}) P_{i n j}=496.8 \mu \mathrm{W}$, and (i,j) $P_{i n}=1083.5$ $\mu \mathrm{W}$. The frequency detuning is $\Delta v=6 \mathrm{GHz}$ and the bias current is $4 \mathrm{~mA}$.

A clear P1 dynamics in the total and orthogonal polarized powers is obtained when increasing $P_{i n j}$ as it is shown in Fig. 4(c). Fig. 4(d) shows that the orthogonal polarization is excited. The frequency difference between the orthogonal polarization and injection frequencies is $6.1 \mathrm{GHz}$ that corresponds to the frequency of the peak in the RF spectrum. This is an indication of a P1 dynamics caused by beating between the optical injection and the orthogonal mode of the VCSEL. The parallel polarization can not be appreciated in the FP spectrum. However the VCSEL has not reached the PS region yet because the parallel polarization is appreciable in the OSA spectrum. We have used the optical spectra obtained with the OSA to depict the PS region in Fig. 3. Our criterion has been the following: PS is achieved when the ratio between the power of the orthogonal and parallel polarizations is higher than $30 \mathrm{~dB}$. The situation in which PS and P1 dynamics is obtained is illustrated in Figs. 4(e-f). The peaks appearing at the RF spectrum have shifted to larger frequency values. An increase of $P_{i n j}$ produces a larger amount of stimulated recombination of carriers and hence a smaller carrier density. In this way the refractive index and the wavelength of the orthogonal polarization increase producing a larger detuning in Fig. 4(f), that is a "frequency pushing" effect. Peaks in the RF spectrum appear at a frequency $(7.1 \mathrm{GHz})$ that approximately correspond to the frequency detuning in the optical spectrum $(6.3 \mathrm{GHz})$, the discrepancy being caused by the resolution of our FP analyzer $(0.6 \mathrm{GHz})$. The frequency pushing effect will be made clearer in the results presented in the next section.

Further increase of $P_{i n j}$ produces a decrease of the amplitude of the peaks in the RF spectrum (see Fig. 4(g)) and also of the peak corresponding to the orthogonal polarization in the optical spectrum (see Fig. 4(h)). Operation in the SL regime is illustrated in Figs. 4(i-j). The orthogonal polarization mode of the VCSEL is stably locked to the optical injection and the RF spectra become flat. This is in fact the criterion that we have used to depict the SL region in Fig. 3. We note that stable injection locking is observed for larger injected powers than those required for PS. This is in contrast with results reported in [13] in which no locking of the fundamental mode was observed for $\Delta v>0$. This is an indication of the different role played by the much larger values of our birefringence parameter when compared to those considered in [13].

Fig. 5 shows the RF and optical spectra for a negative value of the frequency detuning, $\Delta v=-2 \mathrm{GHz}$. The positions in the mapping of the cases analyzed in Fig. 5 are indicated in Fig. 3 with red stars. Figs. 5(a-b) illustrate the behavior obtained for small values of $P_{i n j}$, in such a way that the system is in the P1 regime. RF spectra of both polarizations and total power have peaks at $1.9 \mathrm{GHz}$ frequency and their harmonics. This frequency corresponds to the frequency detuning that is also visible in the left peak in Fig. 5(b): the small shoulder appearing near zero frequency corresponds to the orthogonal polarization mode that is not clearly separated from the injection peak due to the $0.6 \mathrm{GHz}$ resolution of our FP analyzer. Fig. 5(b) also shows that the parallel polarization has appreciable power. In contrast with Fig. 4 both polarization modes have periodic dynamics, as it can be seen in Fig. 5(a). Periodic behavior in both polarizations was also obtained for 850-nm wavelength VCSELs [14]. A torus bifurcation before reaching the locking region was characterized by a limit cycle dynamics of the parallel polarization at the relaxation oscillation frequency and by a wave mixing dynamics between the orthogonal polarization and the optical injection [14]. Figs. 5 (b),(d) do not give clear indications of such a dynamics. The very different values of birefringence parameter and the single polarization mode characteristics of our free-running VCSEL could explain this discrepancy. Increasing $P_{i n j}$ produces an increase of the wavelength of the orthogonal polarization mode in such a way that it approaches the optical injection wavelength. This is shown in Fig. 5(c-d): RF spectra of both polarizations and total power have a major peak located at 1.5 $\mathrm{GHz}$ and the shoulder in the optical spectrum tends to merge with the optical injection peak.

The peaks in the RF spectra tend to disappear as $P_{i n j}$ is further increased as it can be seen in Fig. 5(e). Broad spectra are obtained that are the signature of irregular and possibly chaotic dynamics. This is the behavior characteristic of the IR region in Fig. 3. The better defined peak appears near $1 \mathrm{GHz}$ frequency. This is possibly the value of the frequency detuning that would appear in Fig. 5(f) if our FP analyzer had a better resolution. The amplitude of the peak near zero frequency in Fig. 5(f) has increased due to the merger of the orthogonal 
polarization and the injection peaks. The peak corresponding to the parallel polarization is much weaker and broader than that corresponding to the orthogonal polarization (the inset in Fig. 5(f) is a zoom of that peak). This means that the parallel polarized mode dynamics is more irregular than the dynamics of the orthogonal mode. In fact Fig. 5(e) shows that the RF spectrum corresponding to the parallel polarized power is much broader than that of the orthogonal polarized power. This corresponds to irregular and periodic dynamics for the parallel and orthogonal polarizations, respectively.
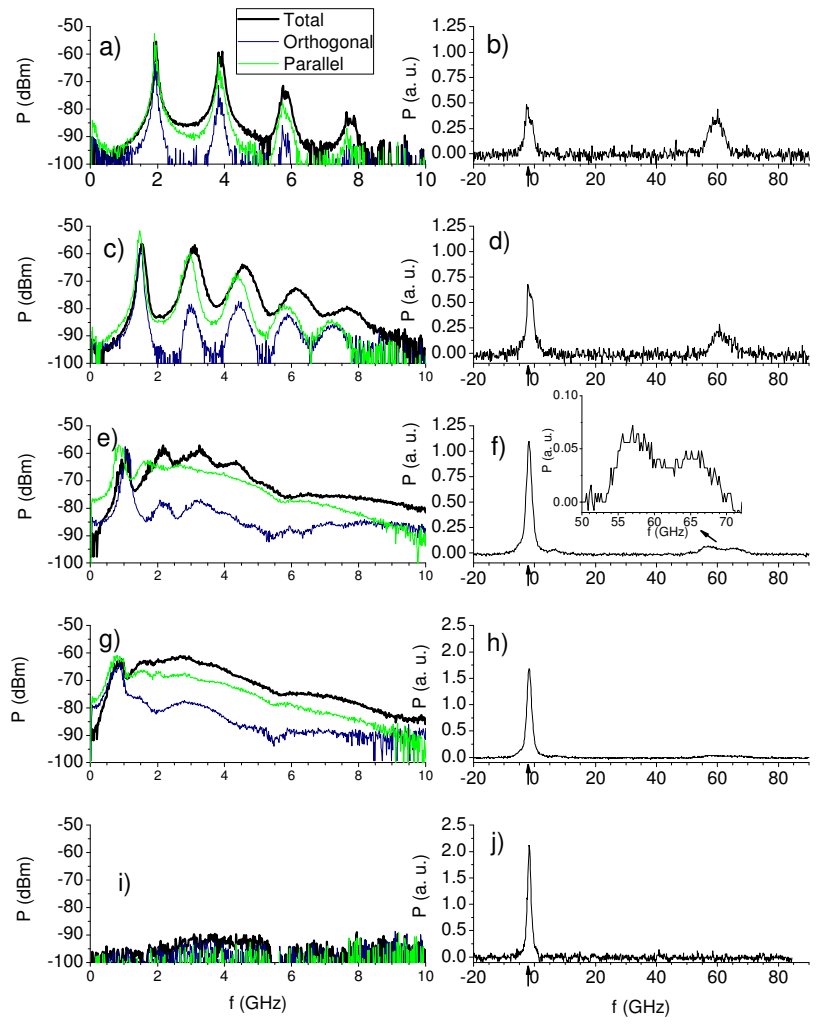

Fig. 5. (color on line) (Left column) RF spectra of the total and polarized powers. (Right column) Optical spectra of the total emitted light. Several values of injected power are considered: $(\mathrm{a}, \mathrm{b}) P_{i n j}=26.9 \mu \mathrm{W},(\mathrm{c}, \mathrm{d}) P_{i n j}=35.5$ $\mu \mathrm{W}$, (e-f) $P_{i n j}=42.4 \mu \mathrm{W}$, (g,h) $P_{i n j}=53.6 \mu \mathrm{W}$, and $(\mathrm{i}, \mathrm{j}) P_{i n j}=79.1 \mu \mathrm{W}$. The frequency detuning is $\Delta v=-2 \mathrm{GHz}$ and the applied bias current is $4 \mathrm{~mA}$.

Figs. 5(g-h) show the spectra when $P_{i n j}$ is increased such that the system is near the border of the IR region in Fig. 3. Peaks in the broad RF spectra are less defined than those in Fig. 5(e). Fig. 5(h) shows that the amplitude of the peak corresponding to the parallel polarization is very small. In fact the PS is obtained by increasing slightly $P_{i n j}$ to $65 \mu \mathrm{W}$. A sudden decrease of the RF spectra to the flat shape characteristic of SL is also obtained at that injected power value. Spectra corresponding to the SL regime are illustrated in Figs. 5(i-j) when $P_{i n j}=79.1 \mu \mathrm{W}$.

One of the main differences between results for positive and negative $\Delta v$ is the following. When $\Delta v$ is positive the only polarization that contributes to the dynamics of the total power is the orthogonal one, as it is shown in the RF spectra of Fig. 4. The parallel polarization is characterized by flat and much weaker RF spectra. When $\Delta v$ is negative, both polarizations contribute to the dynamics of the total power as it is illustrated in the RF spectra of Fig. 5.

\section{NONLINEAR DYNAMICS AT LARGE BIAS CURRENT}

Fig. 6 shows the mapping of the dynamics of the total power when the bias current of the VCSEL subject to orthogonal optical injection is increased to $8 \mathrm{~mA}\left(4.88 I_{t h}\right)$. There are several differences with respect to the mapping discussed in the previous section. First, period doubling (P2) dynamics is obtained for positive and negative $\Delta v$ values. Second, PS can be observed in a periodic dynamical regime including P2 behavior. Third, the PS and the SL regions become more asymmetric as the current is increased, as it was obtained in [27] and [28], respectively. As in Fig. 3, PS is always accompanied with SL for negative $\Delta v$. Also the irregular behavior is only obtained for negative values of $\Delta v$.

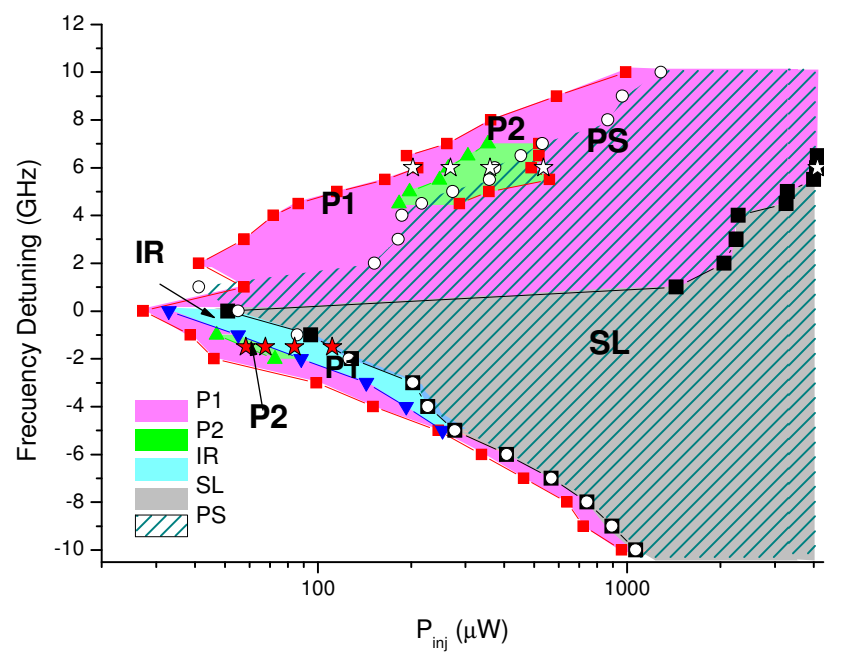

Fig. 6. (color online) Stability map of the VCSEL subject to orthogonal optical injection. Different regions are observed: SL (stable injection locking), P1 (period 1), P2 (period 2), IR (irregular dynamics), and PS (polarization switching). The stars mark the situations analyzed in Figs 7 and 8. Applied bias current of $8 \mathrm{~mA}$.

RF and optical spectra are shown in Fig. 7 for different dynamical regimes. They correspond to a fixed frequency detuning of $6 \mathrm{GHz}$ and different values of $P_{i n j}$. The cases considered in Fig. 7 are identified in Fig. 6 with stars. Fig. 7(a) show the dynamics near the border of the $\mathrm{P} 1$ region. A peak in the RF spectra of the total power and of both linear polarizations appear near $6.4 \mathrm{GHz}$, that is near the difference $(6.1 \mathrm{GHz})$ between the orthogonal polarization and injection frequencies in Fig. 7(b). A smaller symmetric peak is also observed at $-6.1 \mathrm{GHz}$. A period doubling dynamics is illustrated in Fig. 7(c). Two well defined peaks appear at 3.5 and $6.9 \mathrm{GHz}$. These frequencies correspond to the relaxation oscillation frequency of the free-running VCSEL, $v_{R}$, and to $2 v_{R}\left(v_{R}\right.$ measured at $8 \mathrm{~mA}$ is $\left.3.5 \mathrm{GHz}\right)$. This is probably due to a subharmonic resonance. Four peaks appear in the left part of Fig. 7(d). The separation between consecutive peaks is 7.1 $\mathrm{GHz}$. This figure also shows that the parallel polarization is 
still excited with a constant value without contributing to the dynamics of the total power as its RF spectrum is flat and very weak (see Fig. 7(c)).
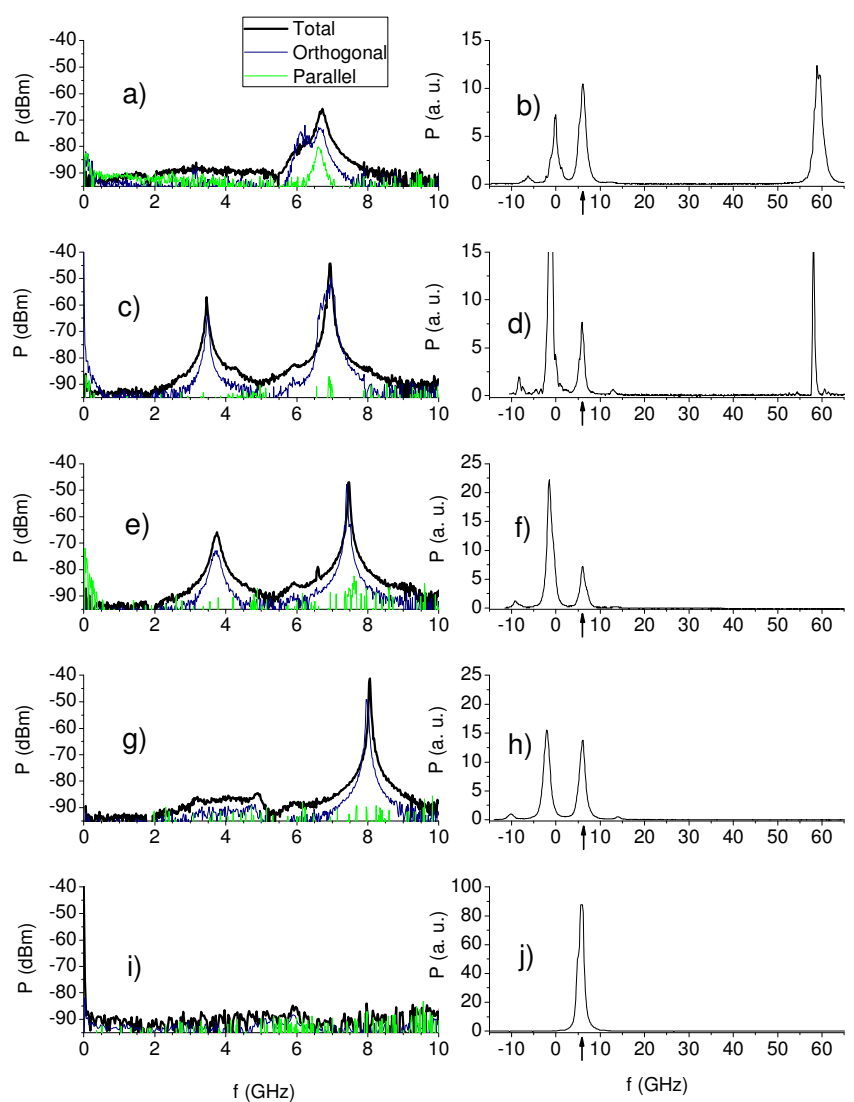

Fig. 7. (color on line) (Left column) RF spectra of the total and polarized powers. (Right column) Optical spectra of the total emitted light. Several values of injected power are considered: $(\mathrm{a}, \mathrm{b}) P_{i n j}=202 \mu \mathrm{W},(\mathrm{c}, \mathrm{d}) P_{i n j}=268.2$ $\mu \mathrm{W}$, (e-f) $P_{i n j}=340.3 \mu \mathrm{W}$, (g,h) $P_{i n j}=535.6 \mu \mathrm{W}$, and (i,j) $P_{\text {inj }}=4120 \mu \mathrm{W}$. The frequency detuning is $\Delta v=6 \mathrm{GHz}$ and the applied bias current is $8 \mathrm{~mA}$.

Figs. 7(e-f) illustrate the spectra when PS is being achieved. Both, the orthogonal polarization and the total power have P2 dynamics as it can be seen in Fig. 7(e). PS is then observed in a periodic dynamical regime including P2 behavior. Further increase of $P_{i n j}$ produces P1 (Figs. 7(g-h)) and SL (Figs. 7(i-j) dynamics.

The frequency pushing effect is made clear from the results of Fig. 7. Frequency differences between the optical injection and the orthogonal polarization peaks are $6.1,7.1,7.3$, and 8 $\mathrm{GHz}$ in Figs. 7(b),(d),(f), and (h), respectively. These results match well with the values of the frequency of the main peak in the RF spectra: 6.4, 6.9, 7.4, and $8 \mathrm{GHz}$ in Figs. 7(a),(c),(e), and $(\mathrm{g})$, respectively. The frequency of the main peak in the RF spectra corresponds to the frequency detuning in the optical spectra in the presence of optical injection.

Fig. 8 shows the RF and optical spectra for a negative $\Delta v$ of $-1.5 \mathrm{GHz}$. White stars in Fig. 6 show the position in the mapping of the cases analyzed in Fig. 8. Figs. 8(a-b) illustrate the P1 dynamics obtained at low values of $P_{i n j}$. RF spectra of both polarizations have peaks at $3 \mathrm{GHz}$ frequency and their harmonics. P2 dynamics is obtained for both linear polarizations when increasing $P_{i n j}$ as it can be seen in Fig. 8(c). Fig. 6 shows that the region in which P2 dynamics appears is very narrow. Figs. 8(a),(c) show that in contrast with the results obtained at positive $\Delta v$ both polarization modes have periodic dynamics. Irregular dynamics in both linear polarizations is observed in Figs. 8(e)-(f). Dynamics seem more irregular than those observed in Fig. $5(\mathrm{~g})$ because peaks are much more smeared, specially those corresponding to the RF spectrum of the orthogonal polarization.
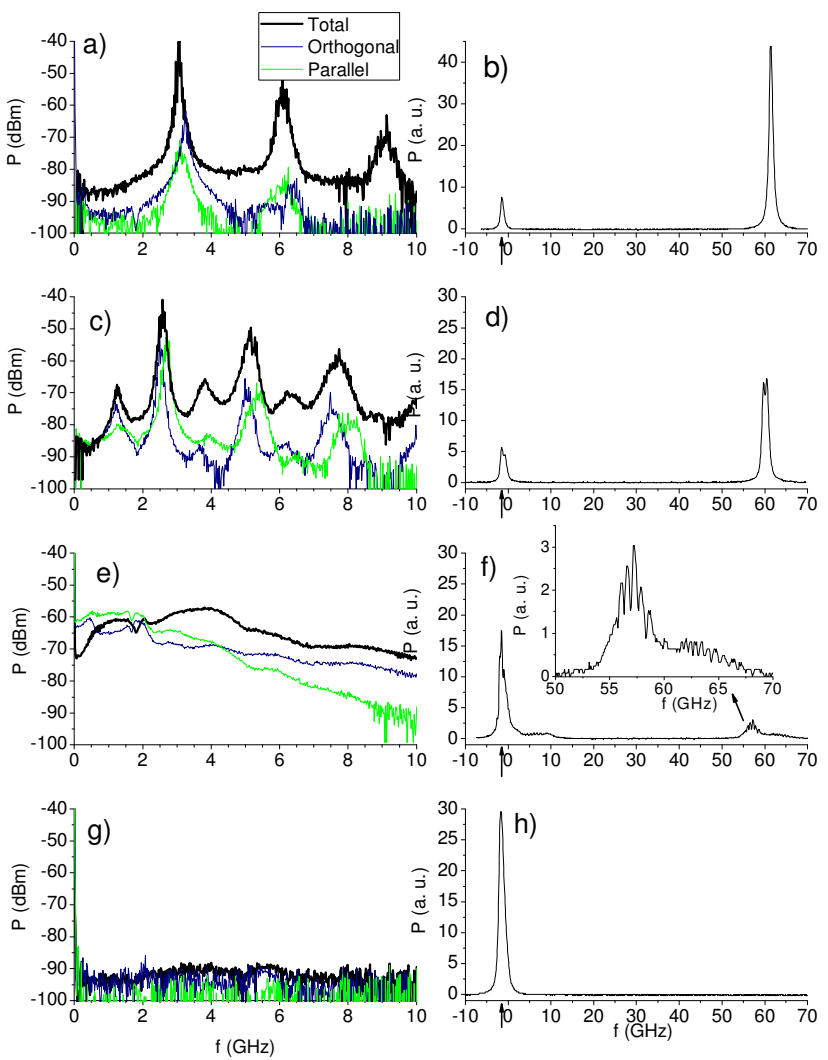

Fig. 8. (color on line) (Left column) RF spectra of the total and polarized powers. (Right column) Optical spectra of the total emitted light. Several values of injected power are considered: $(\mathrm{a}, \mathrm{b}) P_{i n j}=58.5 \mu \mathrm{W},(\mathrm{c}, \mathrm{d}) P_{i n j}=67.6$ $\mu \mathrm{W}$, (e-f) $P_{i n j}=84 \mu \mathrm{W}$, and $(\mathrm{g}, \mathrm{h}) P_{i n j}=111.2 \mu \mathrm{W}$. The frequency detuning is $\Delta v=-1.5 \mathrm{GHz}$ and the applied bias current is $8 \mathrm{~mA}$.

The inset in Fig. 8(f) shows a zoom of the peak corresponding to the parallel polarization in the optical spectrum. This peak is broad (FWHM of $3 \mathrm{GHz}$ ) because of the irregular dynamics of the parallel polarization. Finally, Figs. 8(g-h) show the spectra when simultaneous SL and PS are achieved.

\section{DISCUSSION AND SUMMARY}

In sections III and IV we have found that when $\Delta v>0$ the only polarization that contributes to the dynamics of the total power is the orthogonal one. However when $\Delta v<0$ both polarizations contribute to the dynamics of the total power. A possible explanation for this behavior is the following. Due to the positive value of the linewidth enhancement factor, when the injected power increases the resonance wavelength 
corresponding to the orthogonal polarization approaches (moves away) the injected wavelength when $\Delta v<0(\Delta v>0)$. In this way optical injection is more effective and causes more intense non-linear effects when $\Delta v<0$, like for instance optical bistability [27]. These more intense nonlinear effects can lead to nonlinear dynamics of both polarizations when $\Delta v$ $<0$, despite the large birefringence parameter of our VCSEL. However when $\Delta v>0$ the dynamics of the parallel polarization is not affected due to the less intense non-linear effects and to the large value of the wavelength splitting between linear polarizations. Our VCSEL has a much larger birefringence parameter than those of short-wavelength VCSELs used in previous studies [13-14]. These VCSELs with small birefringence have a much smaller wavelength separation between linear polarizations, and hence the effect of the optical injection is stronger and their nonlinear dynamical behavior is richer [13-14] than in our case.

Some information about the dynamics of both polarizations when $\Delta v<0$ can be inferred from the RF-spectra, shown in Figs. 5(e),(g), at low frequencies. Spectra of both polarizations are much larger than that of the total power at low frequencies. This situation, that is also found in the dynamics of transverse modes in multimode VCSELs, indicates an anti-correlated power dynamics of individual modes [29]. In the same way, results in Figs. 5(e),(g) suggest that the polarization modal dynamics are in antiphase. This behavior is confirmed by measuring at the oscilloscope the time traces of the total power and of the power of both linear polarizations corresponding to Fig. 5(e). These time traces are shown in Fig. 9. We note that the time traces have not been simultaneously recorded because only one high-speed photodetector was available in our experiment. Simultaneous measurements of the time traces of the power of both linear polarizations will be done in future work to distinguish their contribution to the dynamics of the total power. For larger bias current, RF spectra of Fig. 8(e) suggest that antiphase dynamics also occur for the case of the irregular dynamics.

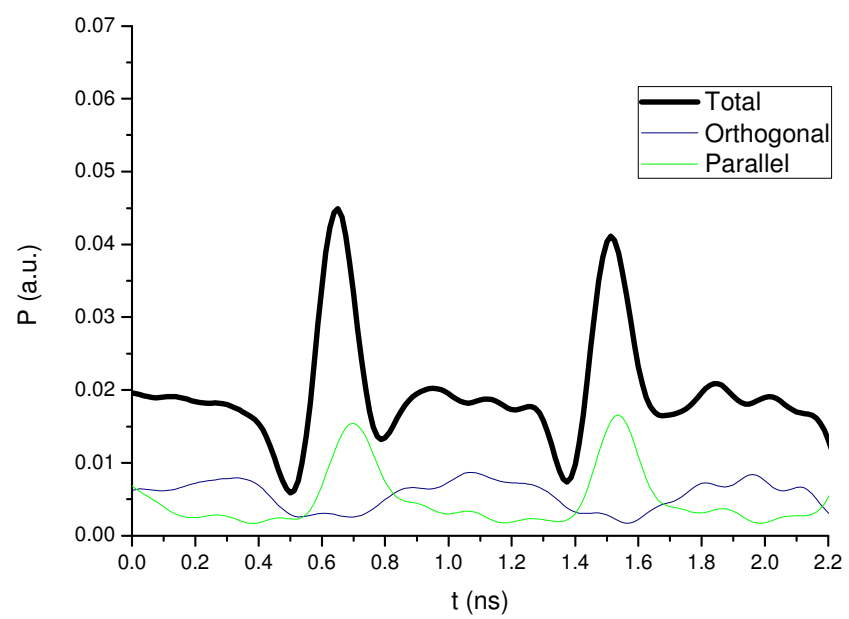

Fig. 9. (color on line) Time traces of the total power and of the power of both linear polarizations. The injected power is $P_{i n j}=42.4 \mu \mathrm{W}$, the frequency detuning is $\Delta v=-2 \mathrm{GHz}$ and the applied bias current is $4 \mathrm{~mA}$.
Summarizing, we have reported the experimental observation of the polarization-resolved nonlinear dynamics of a $1550 \mathrm{~nm}$ single-mode VCSEL subject to orthogonal optical injection. Our VCSEL is characterized by very large values of the birefringence parameter. It is also characterized by emission in a single linear polarization over the whole bias current range in absence of optical injection. Stability maps identifying the boundaries between regions of different dynamics have been measured for several values of the bias current. A rich variety of nonlinear behaviours including periodic, period doubling, and irregular dynamics have been observed for both linear polarizations. More complex dynamics are observed as the bias current is increased. Polarization switching and injection locking induced by the optical injection have been also included in the mapping. Analysis has been made in terms of the frequency detuning, $\Delta v$, between the injected light and the orthogonal linear polarization of the VCSEL. Injection locking has been obtained for larger (similar) injected powers than those corresponding to polarization switching when $\Delta v>1 \mathrm{GHz}(\Delta v$ $<1 \mathrm{GHz})$. When $\Delta v>0$ polarization switching can be observed in a periodic dynamical regime, including both period one and period two behaviors. The only polarization that contributes to the dynamics of the total power is the orthogonal one when $\Delta v>0$. Situations have been described in which the orthogonal polarization have periodic dynamics while the parallel polarization is excited with a constant value. When $\Delta v<0$ both linear polarizations contribute to the dynamics of the total power. Situations have been described in which the parallel polarization has an irregular dynamics while the orthogonal polarization has irregular or periodic dynamics. In other situations both linear polarizations have periodic dynamics. Finally, period doubling has been observed for positive and negative values of $\Delta v$ while irregular dynamics has only been observed for negative values of the frequency detuning.

\section{ACKNOWLEDGEMENTS}

The authors acknowledge Prof. Krassimir Panajotov from Vrije Universiteit Brussels, Dr. Antonio Hurtado and Prof. Mike Adams from the Univ. of Essex (UK) for fruitful discussions. We also acknowledge the reviewers for constructive and clarifying comments.

\section{REFERENCES}

[1] R. Lang, "Injection locking properties of a semiconductor laser," IEEE J. Quantum Electron. vol. 18, no. 6, pp. 976-983, Jun. 1982.

[2] T.B. Simpson, J.M. Liu and A. Gavrielides, "Bandwidth enhancement and broadband noise reduction in injection-locked semiconductor lasers", IEEE Phot. Tech. Lett., vol. 7, no. 7, pp. 709-711, Jul. 1995.

[3] M. J. Adams, A. Hurtado, D. Labukhin, and I. D. Henning, "Nonlinear semiconductor lasers and amplifiers for all-optical information processing”, Chaos, vol. 20, art. 037102, 2010.

[4] F. Koyama, "Recent advances of VCSEL Photonics," J. Lightwave Technol. vol. 24, no. 12, pp. 4502-4513, Dec. 2006.

[5] C. H. Chang, L. Chrostowski, C. J. Chang-Hasnain, "Injection locking of VCSELs," IEEE J. Select. Topics Quantum Electron., vol. 9, no. 5, pp.1386-1393, Sep./Oct 2003.

[6] D. Parekh, X. Zhao, W. Hofmann, M. C. Amann, L. A. Zenteno and C. J. Chang-Hasnain, "Greatly enhanced modulation response of injection- 
locked multimode VCSELs," Opt. Exp., vol. 16, no. 26, pp. 2158221586, Dec. 2008.

[7] D. Parekh, B. Zhang, X. Zhao, Y. Yue, W. Hofmann, M.C. Amann, A. Willner, and C. J. Chang-Hasnain, "Long distance single-mode fiber transmission of multimode VCSELs by injection locking", Opt. Exp., vol. 18, no.20, pp. 20552-20557, 2010.

[8] T. Katayama, T. Ooi, and H. Kawaguchi, "Experimental demonstration of multi-bit optical buffer memory using $1.55-\mu \mathrm{m}$ polarization bistable vertical-cavity surface-emitting lasers", IEEE J. Quantum Electron., vol. 45, no. 11, pp. 1495-1504, Nov. 2009.

[9] S. Wieczorek, B. Krauskopf, T.B. Simpson and D. Lenstra, "The dynamical complexity of optically injected semiconductor lasers." Phys. Rep., vol. 416, no. 1-2, pp. 1-128, Sep. 2005.

[10] H. Li, T. Lucas, J. G. McInerney, M. Wright, and R. A. Morgan, "Injection locking dynamics of vertical cavity semiconductor lasers under conventional and phase conjugate injection, IEEE J. Quantum Electronics, vol. 32, no. 3, pp. 227-235, Feb. 1996.

[11] Y. Hong, P.S. Spencer, P. Rees and K.A. Shore, "Optical injection dynamics of two-mode vertical cavity surface-emitting semiconductor lasers", IEEE J. Quantum Electronics, vol. 38, no. 3, pp. 274-278, Mar. 2002.

[12] Y. Hong, P. S. Spencer, S. Bandyopadhyay, P. Rees, and K. A. Shore, "Polarization resolved chaos and instabilities in a VCSEL subject to optical injection", Opt. Comm., vol. 216, pp. 185-187, 2003.

[13] J. Buesa, I. Gatare, K. Panajotov, H. Thienpont, and M. Sciamanna, "Mapping of the dynamics induced by orthogonal optical injection in vertical-cavity surface-emitting lasers", IEEE J. Quantum Electron., vol. 42, no. 2, pp. 198-207, Feb. 2006.

[14] I. Gatare, M. Sciamanna, M. Nizette, and K. Panajotov, "Bifurcation to polarization switching and locking in vertical-cavity surface-emitting lasers with optical injection", Phys. Rev. A, vol. 76, no. 031803(R), 2007.

[15] A. Valle, I. Gatare, K. Panajotov, and M. Sciamanna, "Transverse mode switching and locking in vertical-cavity surface-emitting lasers subject to orthogonal optical injection", IEEE J. Quantum Electron. vol.43, pp.322-333, Apr. 2007.

[16] I. Gatare, M. Sciamanna, M. Nizette, H. Thienpont and K. Panajotov, "Mapping of two-polarization-mode dynamics in vertical-cavity surface-emitting lasers with optical injection," Phys. Rev. E., vol. 80, no. 026218 , Aug. 2009

[17] A. Hurtado, A. Quirce, A. Valle, L. Pesquera, and M. J. Adams, "Nonlinear dynamics induced by parallel and orthogonal optical injection in $1550 \mathrm{~nm}$ vertical-cavity surface-emitting lasers (VCSELs)", Opt. Exp., vol. 18, no. 9, pp. 9423-9428, 2010.

[18] K. D. Choquette, R. P. Schneider, K. L. Lear, and R. E. Leibenguth, "Gain-dependent polarization properties of vertical-cavity lasers", IEEE J. Sel. Topics in Quantum Electron., vol. 1, pp. 661-666, Jun. 1995.

[19] B. Ryvkin, K. Panajotov, A. Georgievski, J. Danckaert, M. Peeters, G, Verschaffelt, H. Thienpont, and I. Veretennicoff, "Effect of photon energy-dependent loss and gain mechanisms on polarization switching in vertical-cavity surface-emitting lasers", J. Opt. Soc. Amer. B, vol. 16, pp. 2106-2113, 1999.

[20] A. Valle, L. Pesquera, and K. A. Shore, "Polarization behaviour of birefringent multitransverse mode vertical-cavity surface-emitting lasers", IEEE Photon. Technol. Lett., vol. 9, no. 5, pp. 557-559, May 1997.

[21] J. M. Martín-Regalado, F. Prati, M. San Miguel, and N. B. Abraham, "Polarization properties of vertical-cavity surface-emitting lasers", IEEE J. Quantum Electron, vol. 33, pp. 765-783, May 1997.

[22] M. P. Van Exter, M. B. Willemsen, and J. P. Woerdman, "Polarization fluctuations in vertical-cavity semiconductor lasers", Phys. Rev. A, vol. 58, pp. 4191-4205, 1998.

[23] Z. G. Pan, S. Jiang, M. Dagenais, R. A. Morgan, K. Kojima, M. T. Asom, and R. E. Leibenguth, "Optical injection induced polarization bistability in vertical-cavity surface-emitting lasers," Appl. Phys. Lett., vol. 63, no. 22, pp. 2999-3001, Nov.1993.

[24] K. H. Jeong, K. H. Kim, S. H. Lee, M. H. Lee, B. S. Yoo and K. A. Shore, "Optical injection-induced polarization switching dynamics in $1.5 \mu \mathrm{m}$ wavelength single-mode vertical-cavity surface-emitting lasers," IEEE Photon. Technol. Lett., vol. 20, no. 9-12, pp. 779-781, May. 2008.

[25] A. Hurtado, I. D. Henning, and M. J. Adams, "Two-wavelength switching with a $1550 \mathrm{~nm}$ VCSEL under single orthogonal optical injection," IEEE J. Sel. Top. in Quantum Electron, vol. 14, no. 3, pp. 911-917, May/Jun.2008.

[26] A. Valle, M. Gomez-Molina, and L. Pesquera, "Polarization bistability in $1550 \mathrm{~nm}$ wavelength single-mode vertical-cavity surface-emitting lasers subject to orthogonal optical injection," IEEE J. Sel. Top in Quantum Electron., vol. 14, no. 3, pp. 895-902, May/Jun.2008.

[27] M. S. Torre, A. Hurtado, A. Quirce, A. Valle, L. Pesquera, and M. J. Adams, "Polarization switching in long-wavelength VCSELs subject to orthogonal optical injection", IEEE J. Quantum Electron., vol. 47, no. 1, pp. 92-99, Jan. 2011.

[28] N. Khan, K. Schires, A. Hurtado, I. D. Henning, and M. J. Adams, "Current dependence of polarization switching and locking in an optically injected $1550 \mathrm{~nm}$ VCSEL", IET Optoelectronics. To appear.

[29] A. Valle, L. Pesquera, "Relative intensity noise of multitransverse-mode vertical-cavity surface-emitting lasers", IEEE Photon. Technol. Lett., vol. 13, no. 4, pp. 272-274, Apr. 2001.

P. Pérez received the Licenciado en Física (Ms. Sc.) degree from the Universidad de Cantabria, Spain, in 2010. He is currently working toward the $\mathrm{PhD}$ degree in Physics. His current research interests are in the areas of dynamics of vertical-cavity surface-emitting lasers and optical injection effects in semiconductor lasers.

A. Quirce received the Licenciada en Física (Ms. Sc.) degree from the Universidad de Cantabria, Spain, in 2008. She is currently working toward the $\mathrm{PhD}$ degree in Physics. Her current research interests are in the areas of dynamics of vertical-cavity surface-emitting lasers and optical injection effects in semiconductor lasers.

L. Pesquera was born in Vega de Infanzones, León, Spain, in 1952. He received the M.Sc. degree in physics in 1974 from the Universidad de Valladolid, Spain. He was a postgraduate fellow at the Université de Paris VI during 1977-1980. He received the Ph.D. degree in physics in 1980 from the Universidad de Cantabria, Santander, Spain.

In 1980 he joined the Departamento de Física Moderna of the Universidad de Cantabria. Since 1991 he has been Professor of Physics at the Universidad de Cantabria. In 1995 he joined the Instituto de Física de Cantabria (CSICUC). His research work started in the field of stochastic processes applied to Physics and he has made contributions to the foundations of quantum physics, fluctuations in nuclear reactors, disordered systems and laser physics. $\mathrm{He}$ is currently working on the modeling of noise and nonlinear properties of semiconductor lasers and their applications to optical communication systems.

A. Valle was born in Reinosa, Cantabria, Spain, in 1965. He received the M. Sc. and Ph.D. degree in physics from the Universidad de Cantabria, Spain, in 1988 and 1993, respectively.

During 1994 and 1995, he was a postdoctoral fellow at the School of Electronic and Electrical Engineering at the University of Bath, England. In 1996 he joined the Instituto de Física de Cantabria (CSIC-UC). Since 1998 he has been lecturer at the Departamento de Física Moderna at the University of Cantabria, Spain. His research interests are in the areas of vertical-cavity surface-emitting lasers, noise and nonlinear dynamics of semiconductor lasers. 